\title{
Results of Long-Term Carcinogenicity Bioassays on Coca-Cola Administered to Sprague-Dawley Rats
}

\author{
FIORELLA BELPOGGI, MORANDO SOFFRITTI, EVA TIBALDI, \\ LAURA FALCIONI, LUCIANO BUA, AND FRANCESCA TRABUCCO
}

\author{
Cesare Maltoni Cancer Research Center, European Foundation \\ for Oncology and Environmental Sciences "B. Ramazzini," 40010 Bentivoglio, \\ Bologna, Italy
}

\begin{abstract}
Coca-Cola was invented in May 1886 in Atlanta, Georgia by a pharmacist who, by accident or design, mixed carbonated water with the syrup of sugar, phosphoric acid, caffeine, and other natural flavors to create what is known as "the world's favorite soft drink." Coca-Cola is currently sold in more than 200 countries and in early 2000, the company sold its 10 billionth unit case of Coca-Cola branded products. Given the worldwide consumption of Coca-Cola, a project of experimental bioassays to study its long-term effects when administered as substitute for drinking water on male and female Sprague-Dawley rats was planned and executed. The objective of the project was to study whether and how long-term consumption of Coca-Cola affects the basic tumorigram of test animals. The bioassays were performed on rats beginning at different ages, namely: $(a)$ on males and females exposed since embryonic life or from 7 weeks of age; and $(b)$ on males and females exposed from 30, 39, or 55 weeks of age. Overall, the project included 1999 rats. During the biophase, data were collected on fluid and feed consumption, body weight, and survival. Animals were kept under observation until spontaneous death and underwent complete necropsy. The results indicate: (a) an increase in body weight in all treated animals; (b) a statistically significant increase of the incidence in females, both breeders and offspring, bearing malignant mammary tumors; $(c)$ a statistically significant increase in the incidence of exocrine ademonas of the pancreas in both male and female breeders and offspring; and $(d)$ an increased incidence, albeit not statistically significant, of pancreatic islet cell carcinomas in females, a malignant tumor which occurs very rarely in our historical controls. On the basis of the results of this study, excessive consumption
\end{abstract}

Address for correspondence: Morando Soffritti, M.D., Cesare Maltoni Cancer Research Center, European Ramazzini Foundation, Castello di Bentivoglio, 40010 Bentivoglio (BO), Italy. Voice: +39051-6640460; fax: +39-051-6640223.

e-mail: crcfr@ramazzini.it; www.ramazzini.it

Funding for this research was provided entirely by the European Foundation on Oncology and Environmental Sciences "B. Ramazzini."

Ann. N.Y. Acad. Sci. 1076: 736-752 (2006). (C) 2006 New York Academy of Sciences. doi: 10.1196/annals.1371.078 


\section{of regular soft-drinks should be generally discouraged, in particular for children and adolescents.}

KEYwORDS: Coca-Cola; carcinogenicity; long-term bioassay; rat

\section{INTRODUCTION}

Cancer is one the principal public health problems faced by industrialized countries, both because of its epidemiological dimension and because of the growing environmental influences behind its increase to today's epidemic proportions. Diet is often cited as an important factor in the occurrence of cancer; however, influence of diet on health cannot be easily isolated or quantified given the varied composition of the average diet over a lifetime and the complex mixture of substances present in food. While the overall diet is difficult to analyze, specific nutrients and food components have been implicated both in causing cancer and as protective factors in the carcinogenic process.

There are several sources of food components believed to induce cancer. One is the presence of naturally occurring substances, such as aflatoxin found in groundnuts and corn. Another potential source of risk are substances which are added directly to food in order to improve its quality, taste, or stability.

It has long been suggested that total calorie intake may have an important effect on the incidence of cancer. Data by Tannenbaum in the 1940s and 1950 s demonstrated that underfed mice developed fewer spontaneous mammary tumors, primary lung adenomas, and chemically induced tumors. ${ }^{1,2}$ In addition, human obesity has been identified as a risk factor for some types of cancer. ${ }^{3,4}$

Long-term carcinogenicity bioassays on rodents, which reproduce exposure situations experienced by humans as much as possible, is in our opinion, a good approach to study the influence of dietary products on the incidence of spontaneous tumors in a controlled environment. ${ }^{5,6}$ In an attempt to evaluate the interaction between one such product and tumor incidence in rodents, an experimental carcinogenicity bioassay was performed at the Cesare Maltoni Cancer Research Center (CMCRC) of the European Ramazzini Foundation (ERF). The product tested is a beverage mixture which is both caloric and widely consumed in most of the world-Coca-Cola.

Coca-Cola was invented in Atlanta, Georgia, on May 8, 1886 by pharmacist John Pemberton. The regular cola beverage contains water, sugar (about 10\% by weight), carbon dioxide, caramel coloring, acidulates, flavoring substances, and caffeine, all of which are approved for use around the world. Today CocaCola, recognized as the world's number one soft drink brand, is currently sold in more than 200 countries. In 2001, the company sold its 10 billionth unit case of Coca-Cola branded products. ${ }^{7}$ 
The experimental project described in this article was designed to evaluate the long-term effects of Coca-Cola on the spontaneous development of tumors when administered as a substitute for drinking water to Sprague-Dawley rats for the life span.

Since it is known that the age of the animals at the start of the experiment may affect the possible modulating effects of the test compounds, the project includes experiments performed on groups of male and female breeder rats $(30,39$, and 55 weeks old at the start of the experiment), all the offspring of each litter descending from the aforementioned breeders (exposed since intrauterine life), and one group exposed beginning at 7 weeks of age.

\section{MATERIALS AND METHODS}

The Coca-Cola used was supplied by an Italian reseller, in 1-L glass bottles delivered every 2-3 weeks. During the experiments, the bottles were stored at a room temperature of $22^{\circ} \pm 3^{\circ} \mathrm{C}$. Before administering the Coca-Cola to the rodents, the $\mathrm{CO}_{2}$ was first eliminated by mechanical shaking for 60 minutes.

The male (M) and female (F) Sprague-Dawley rats used in this experiment were bred from the colony used at the CMCRC/ERF laboratories for nearly 30 years. Extensive historical data on more than 15,000 rodents are available on the tumor incidence among untreated rats.

At 4 weeks of age, the breeders were identified by ear punch, separated by sex, and assigned to experimental groups so as to have no more than one male and one female from each litter in the same group. Rats were housed 5 per cage until the time of mating, at which time parents were placed in breeding cages (one male with one female). After 7 days, males were re-housed 5 per cage and females were housed individually. After 8 weeks, the females were also re-housed 5 per cage.

All available pups of all litters from treated and untreated dams were included in the various groups of offspring. After weaning (at 4-5 weeks), offspring were identified by ear punch, weighed, separated by sex, and assigned sequentially, litter by litter, to the exposed and control group, respectively. The two groups of 7-week-old rats were randomized in order to have no more than one male and one female from each litter in the same group.

Animals were housed in makrolon cages $(41 \mathrm{~cm} \times 25 \mathrm{~cm} \times 15 \mathrm{~cm})$ with stainless-steel wire tops and a shallow layer of white wood shavings as bedding. Cages were kept in rooms used exclusively for this experiment at a temperature of $21^{\circ} \pm 2{ }^{\circ} \mathrm{C}$ and relative humidity of 50-60\%. A light-dark cycle of 12 hours was maintained using both natural and artificial light sources.

Animals were given the standard Corticella pellet diet (Corticella S.p.A., Bologna, Italy), analyzed for nutritional components and possible contaminants. Every 24 hours, Coca-Cola or drinking water (control) were disposed of and the bottles were cleaned and refilled. 
The animals were checked three times a day from Monday to Saturday, and twice on Sundays and holidays for clinical symptoms and behavior.

Coca-Cola was administered ad libitum instead of drinking water to groups of breeders (55 - 110 per sex per group) 30,39 , or 55 weeks old at the start of the experiment, to their offspring exposed since intrauterine life (24-110 per sex per group), and to a group of 80 males and 80 females, 7 weeks old at the start of the experiment. The administration of Coca-Cola lasted until spontaneous death. Equivalent groups of breeders and offspring administered drinking water served as controls. The entire project involved 1999 rodents.

Mean daily drinking water and feed consumption were measured on the day prior to the start of the breeders' treatment, after 7 days of treatment before mating and then, post-weaning, once weekly for the first 13 weeks from the start of the experiment, then every 2 weeks until 104 weeks. Individual animal weight of breeders and of offspring after weaning was measured once weekly for the first 13 weeks, every 2 weeks until 104 weeks, and then every 8 weeks until the end of the experiments. In order to detect and register all gross lesions, the animals were examined every week for the first 13 weeks, and then every 2 weeks until the end of the experiment.

Upon death, all animals underwent complete necropsy. Histopathology was routinely performed on all macroscopically observed pathological lesions (with a margin of surrounding normal tissue) and on skin and subcutaneous tissue, the brain, pituitary gland, Zymbal glands, salivary glands, Harderian glands, cranium (with oral and nasal cavities and external and internal ear ducts, 5 levels), tongue, thyroid and parathyroid, pharynx, larynx, thymus and mediastinal lymph nodes, trachea, lung and mainstem bronchi, heart, diaphragm, liver, spleen, pancreas, kidneys and adrenal glands, esophagus, stomach (fore and glandular), intestine (4 levels), bladder, prostate, uterus, gonads, interscapular fat pad, subcutaneous and mesenteric lymph nodes, and any other organ or tissue with pathological lesions.

All organs and tissues were preserved in $70 \%$ ethyl alcohol, except for the bones which were fixed in $10 \%$ formalin and then decalcified with $10 \%$ formaldehyde and $20 \%$ formic acid in water solution. The normal specimens were trimmed, following Standard Operating Procedures (SOP) at the CMCRC/ERF laboratories. Trimmed specimens were processed as paraffin blocks, and 3-5 $\mu \mathrm{m}$ sections of every specimen were obtained. Sections were routinely stained with hematoxylin-eosin. Specific stainings were performed when needed. All slides were examined microscopically by the same group of pathologists; a senior pathologist reviewed all tumors and any other lesion of oncological interest.

Statistical analysis was performed using the $\chi^{2}$ test in order to evaluate the significance in tumor incidence differences between treated and control groups. 


\section{RESULTS}

In this article, we present the results of data aggregated from all breeders and all offspring. Offspring include both rodents whose treatment began during embryonic life and those rodents whose treatment began at 7 weeks of age.

A treatment-related difference in fluid consumption was observed, in both sexes of breeders and offspring, with treated animals consuming more than two times as much fluid compared to controls. An opposite treatment-related difference in food consumption was observed in both sexes of breeders and offspring during the experiment, with treated animals consuming about $40 \%$ less feed compared to controls.

An increase in body weight was observed, in both breeders and offspring, among both sexes in treated groups compared to the controls.

No differences in survival were observed among treated males and females breeders compared to the controls. A slight decrease in survival was observed in female offspring compared to the controls.

The occurrence of benign and malignant tumors among male and female rats is shown in TABLE 1 . The differences observed among treated and control animals were as follows:

1. a slight increase in the incidence of malignant tumor-bearing animals was observed in female breeders compared to controls (TABLE 2);

2. a statistically significant increase in females bearing malignant mammary tumors and total malignant mammary tumors, both among breeders $(P$ $<0.01)$ and offspring $(P<0.01)$, compared to the controls (TABLE 3 );

3 . a statistically significant increased incidence in animals bearing adenomas of the exocrine component of the pancreas was observed among male $(P<0.01)$ and female $(P<0.05)$ breeders and male $(P<0.01)$ and female $(P<0.01)$ offspring compared to the controls. No exocrine carcinomas were observed (TABLE 4). Concerning the incidence of islet cell adenomas, no differences were observed in treated breeders or offspring of either sex compared to the controls. An increased incidence of islet cell carcinomas was observed in treated female breeders and treated female offspring compared to the controls. Only one case of carcinoma was observed in treated male offspring. It must be noted that in our historical controls, we observed only $1(0.04 \%)$ islet cell carcinoma out of 2274 untreated females and $5(0.2 \%)$ islet cell carcinomas out of 2265 males.

\section{CONCLUSIONS}

Although Coca-Cola is one of the most consumed soft-drinks worldwide, to our knowledge this study represents the first time that a long-term 


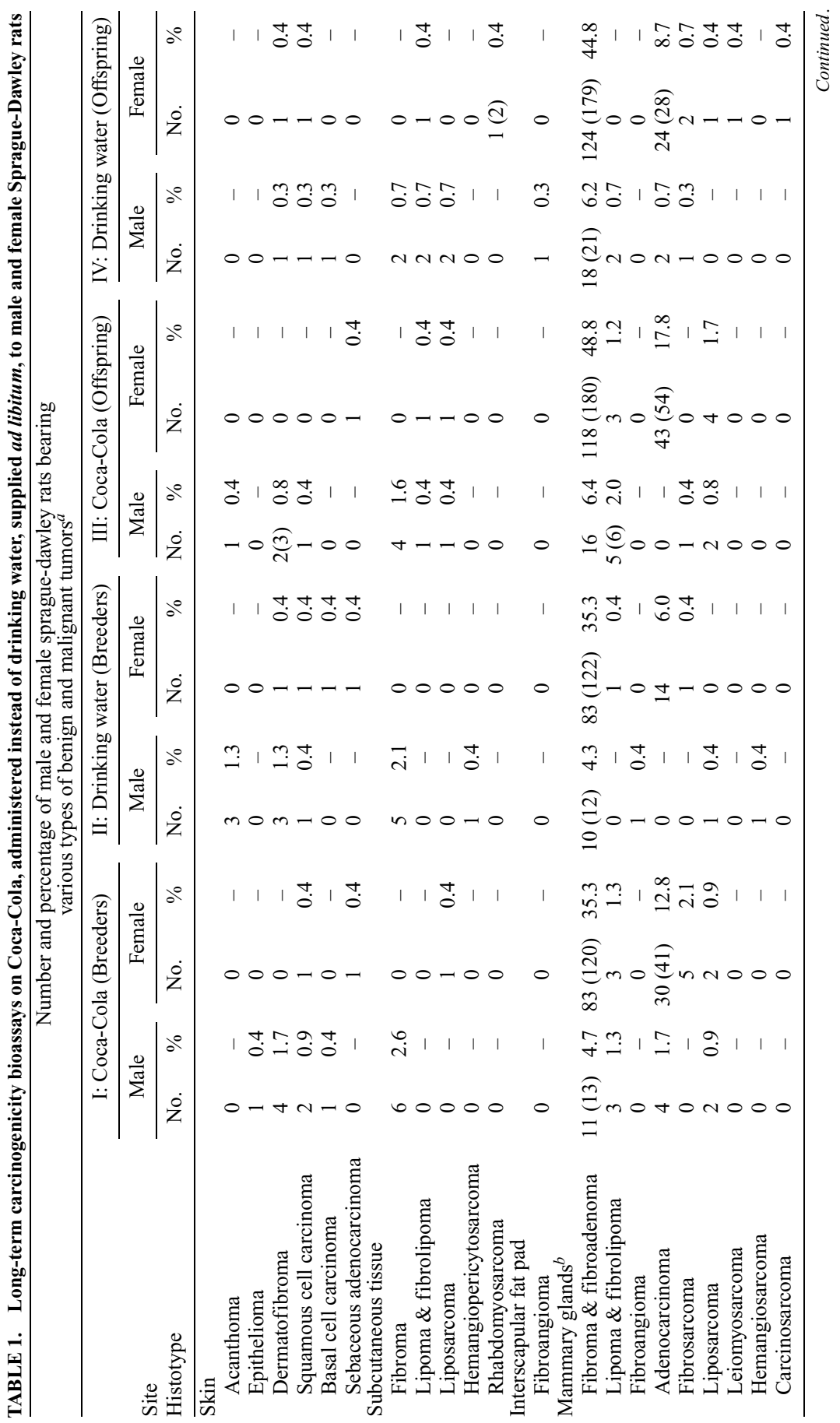




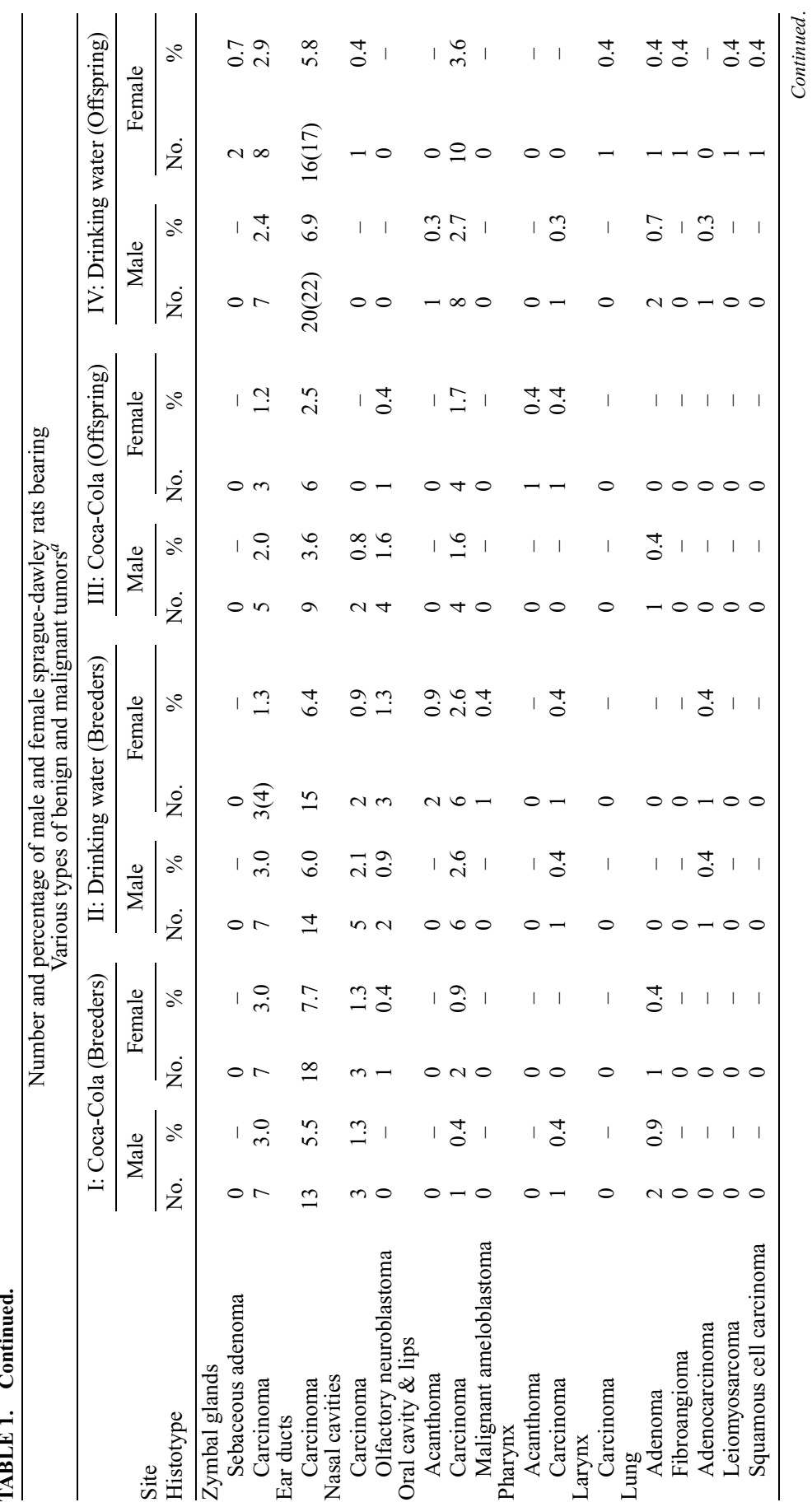




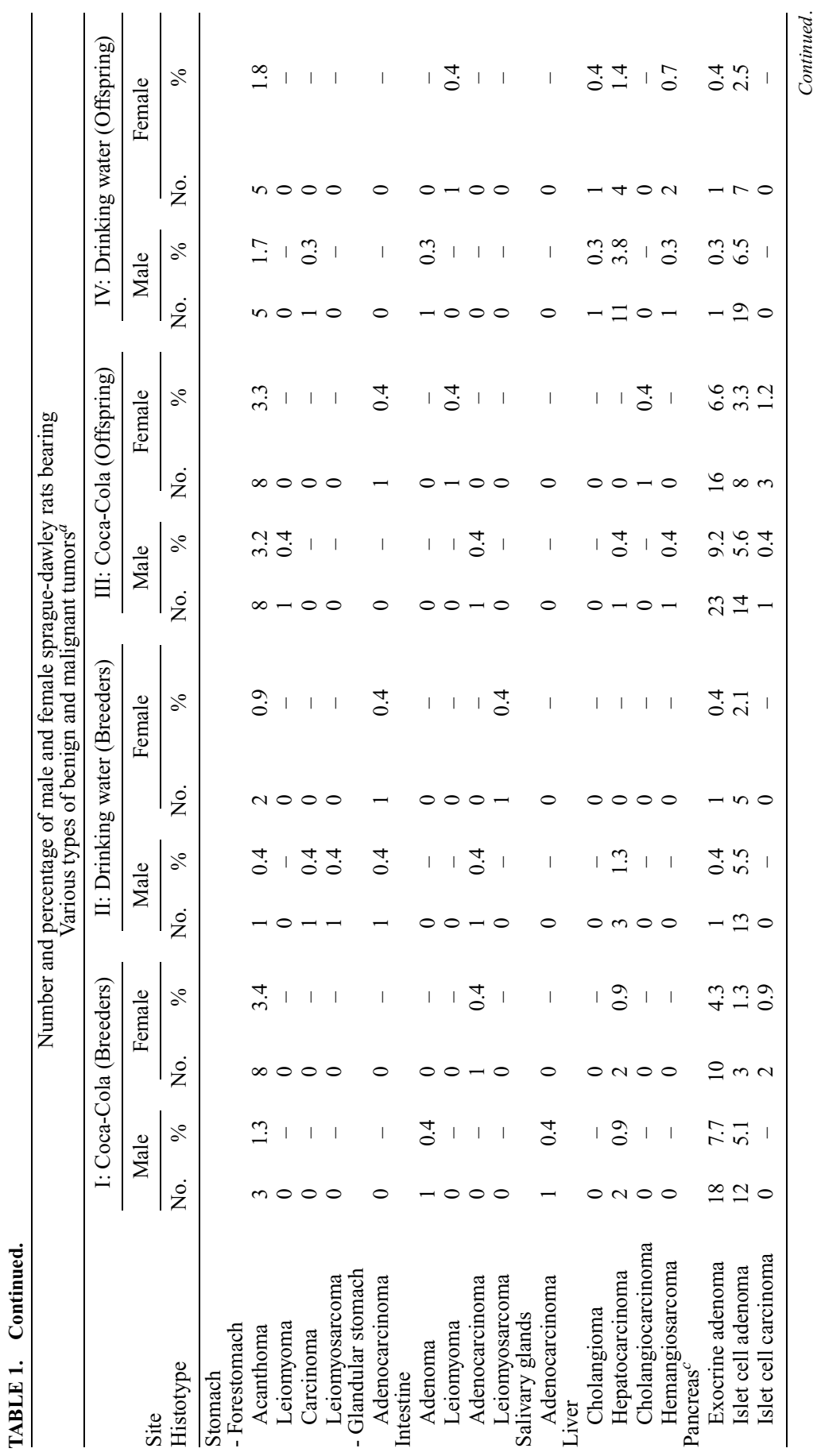




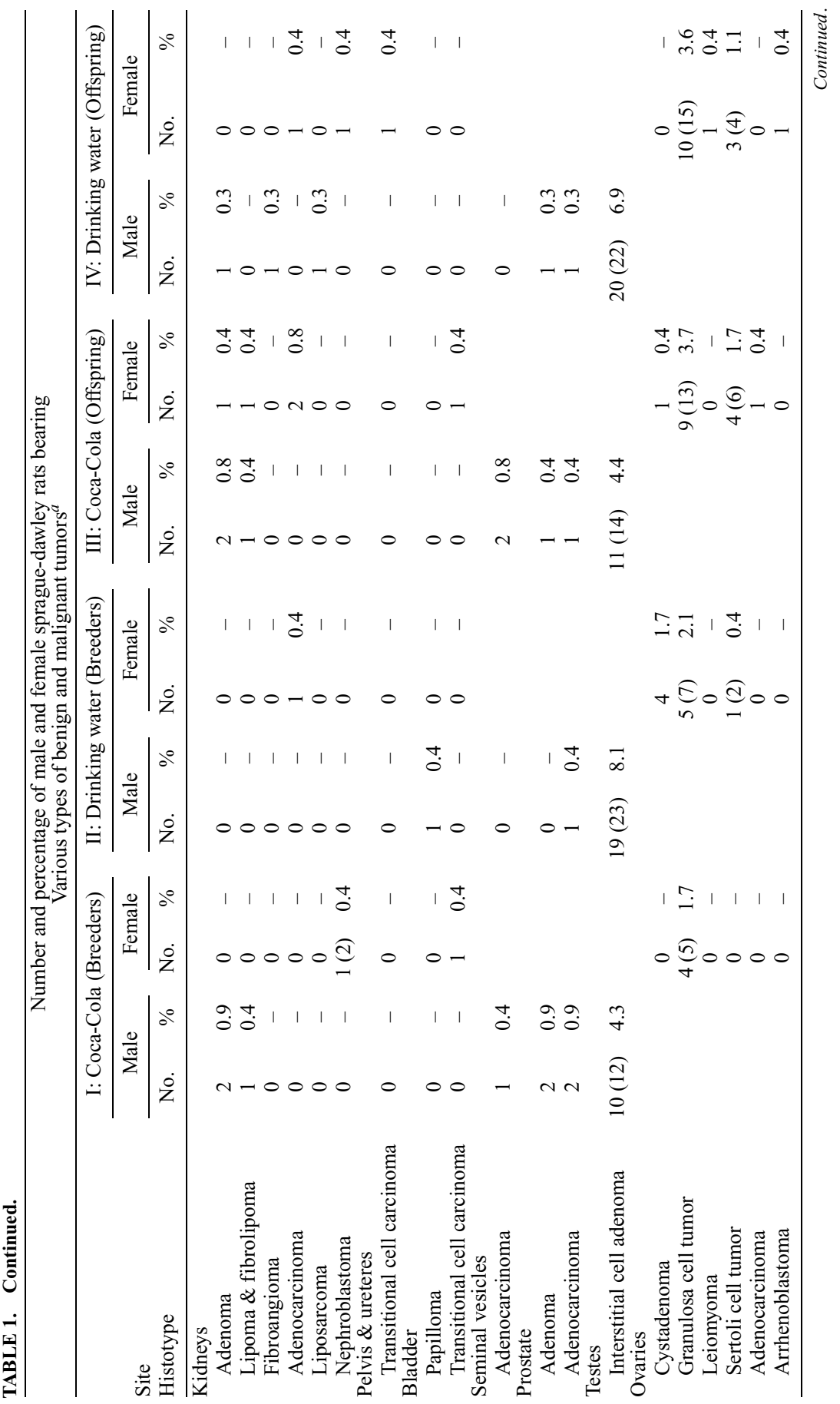




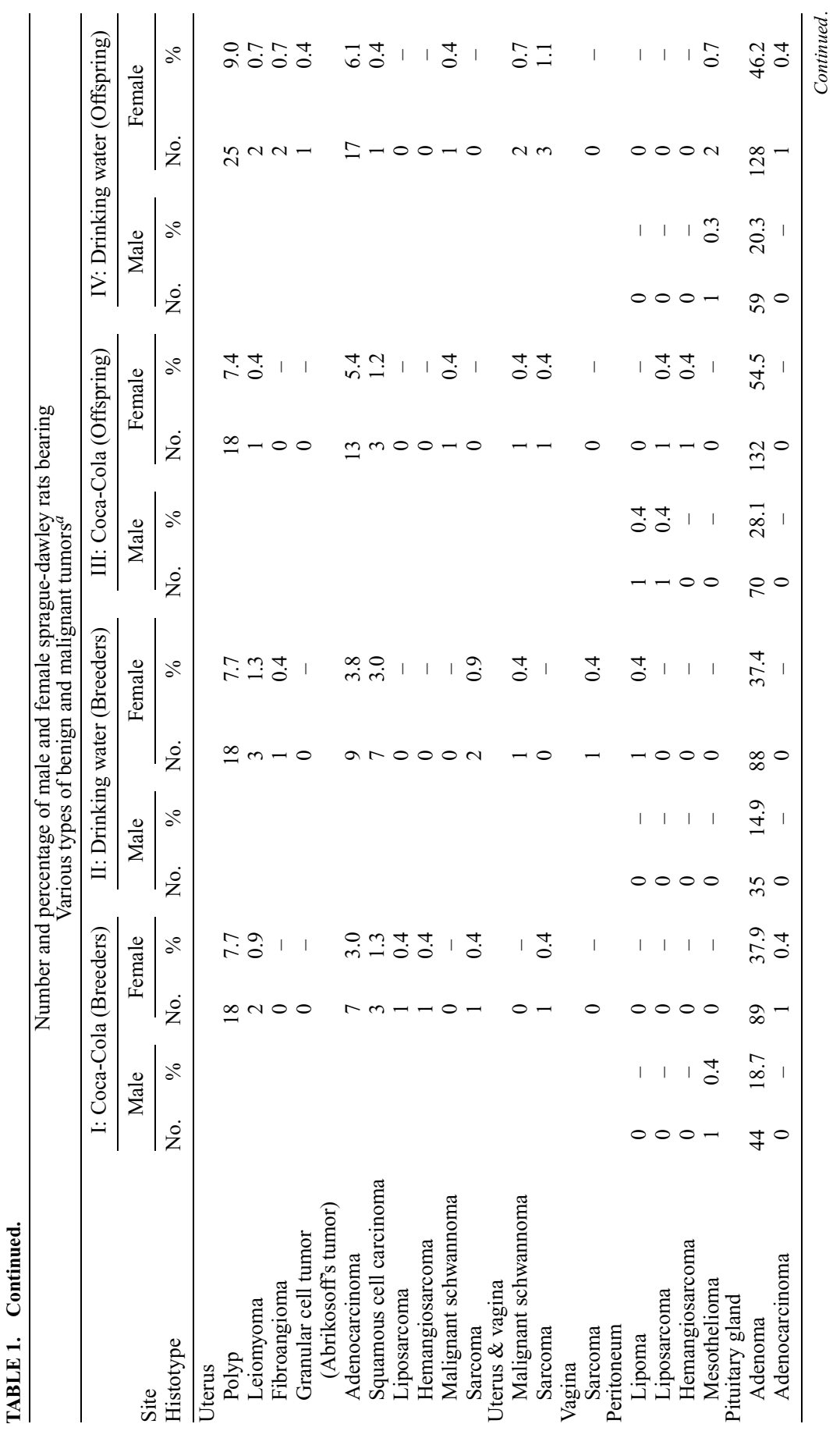




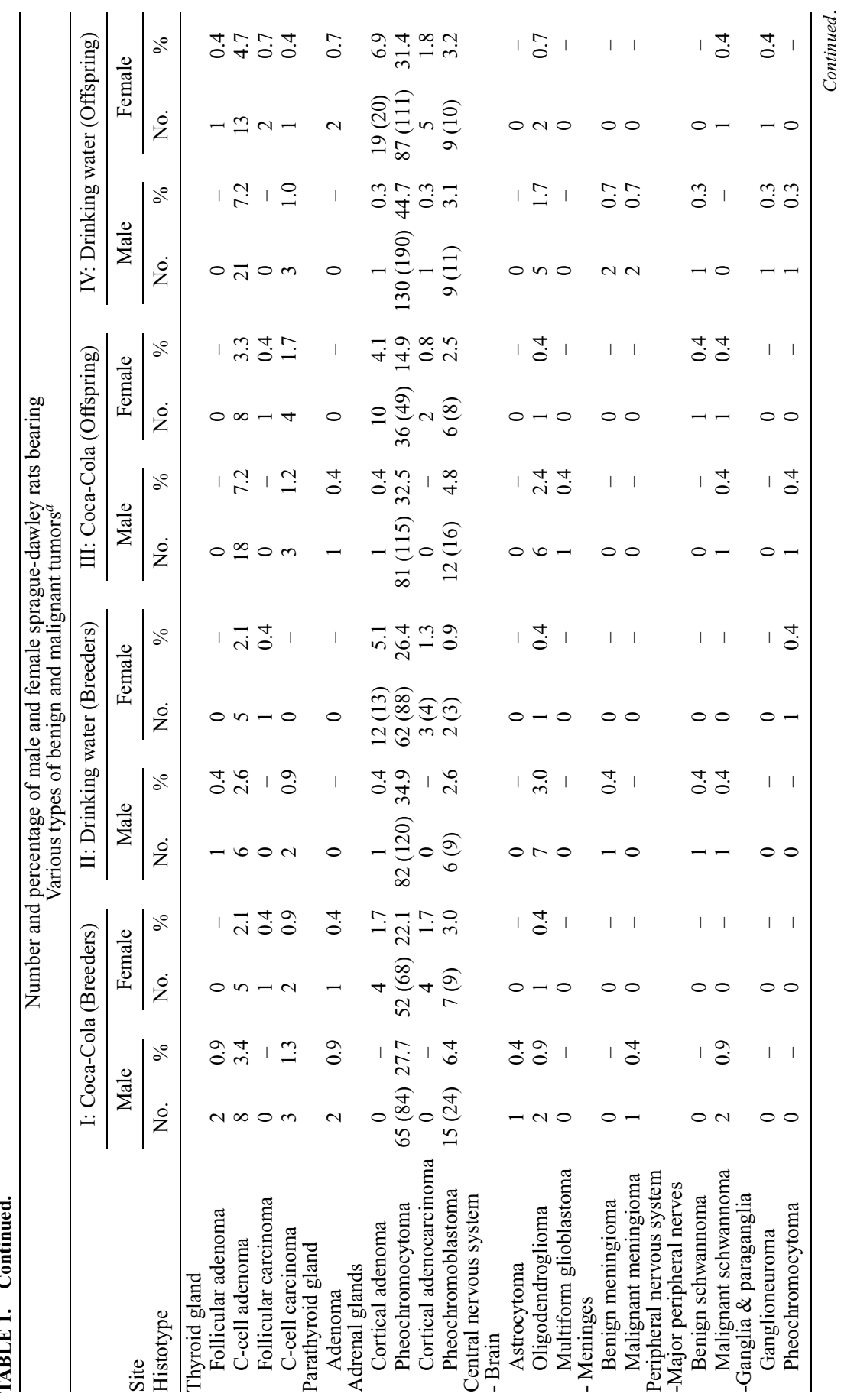




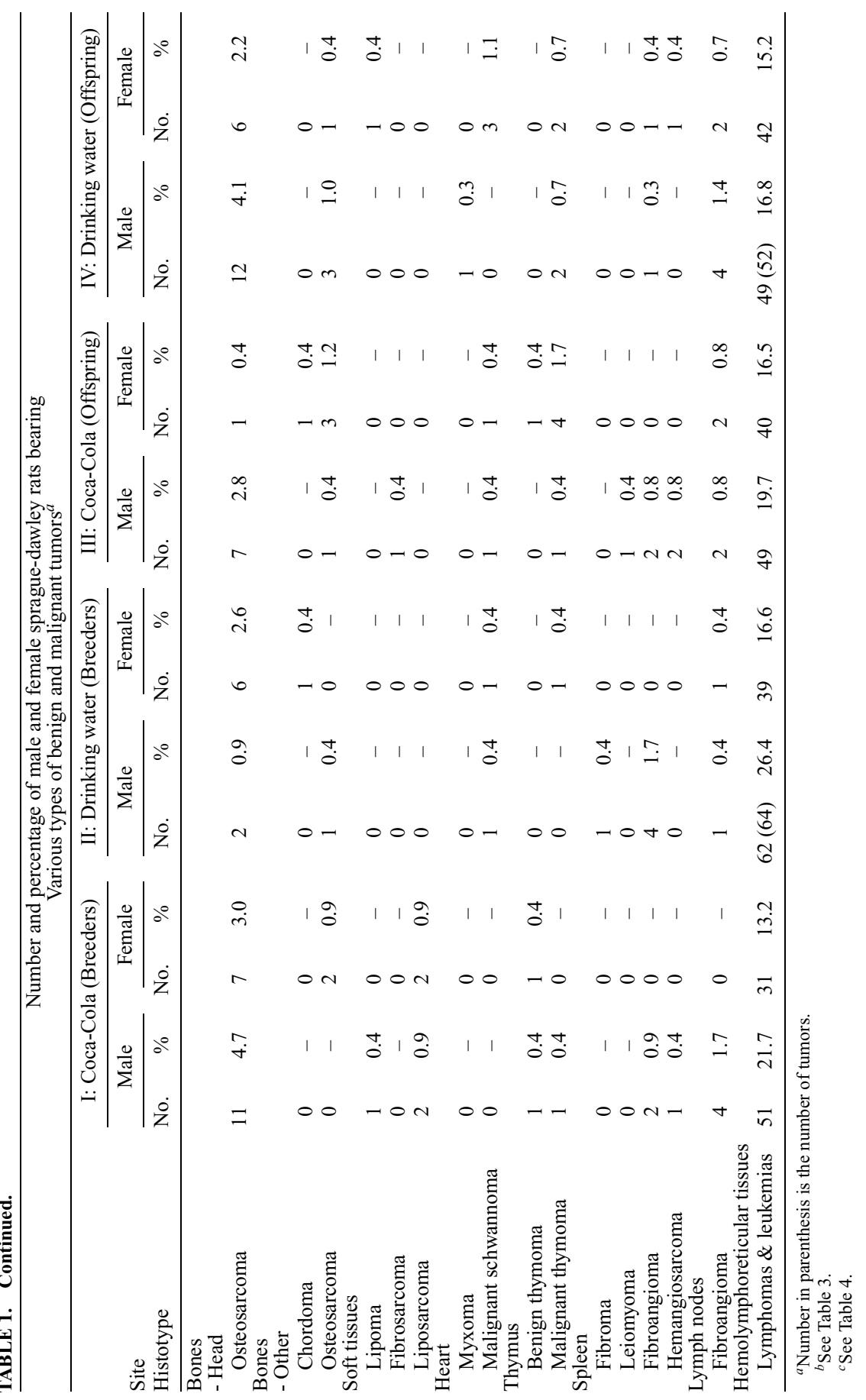




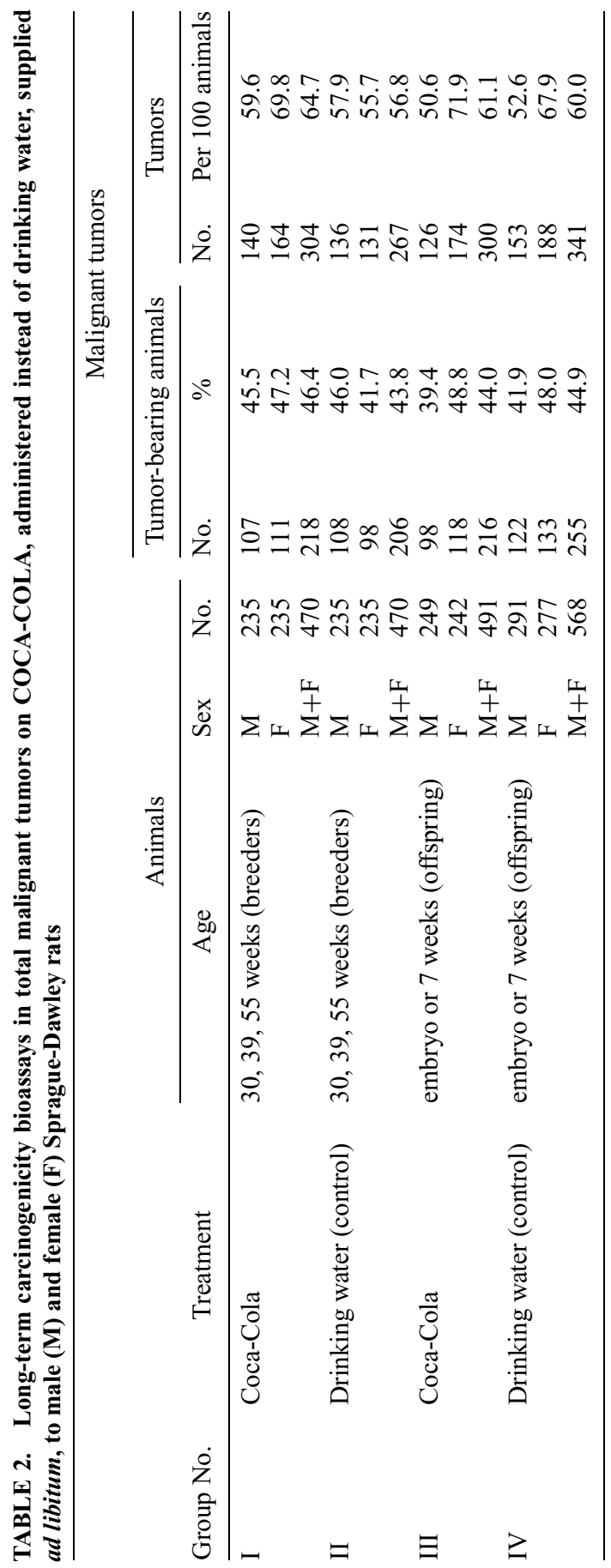




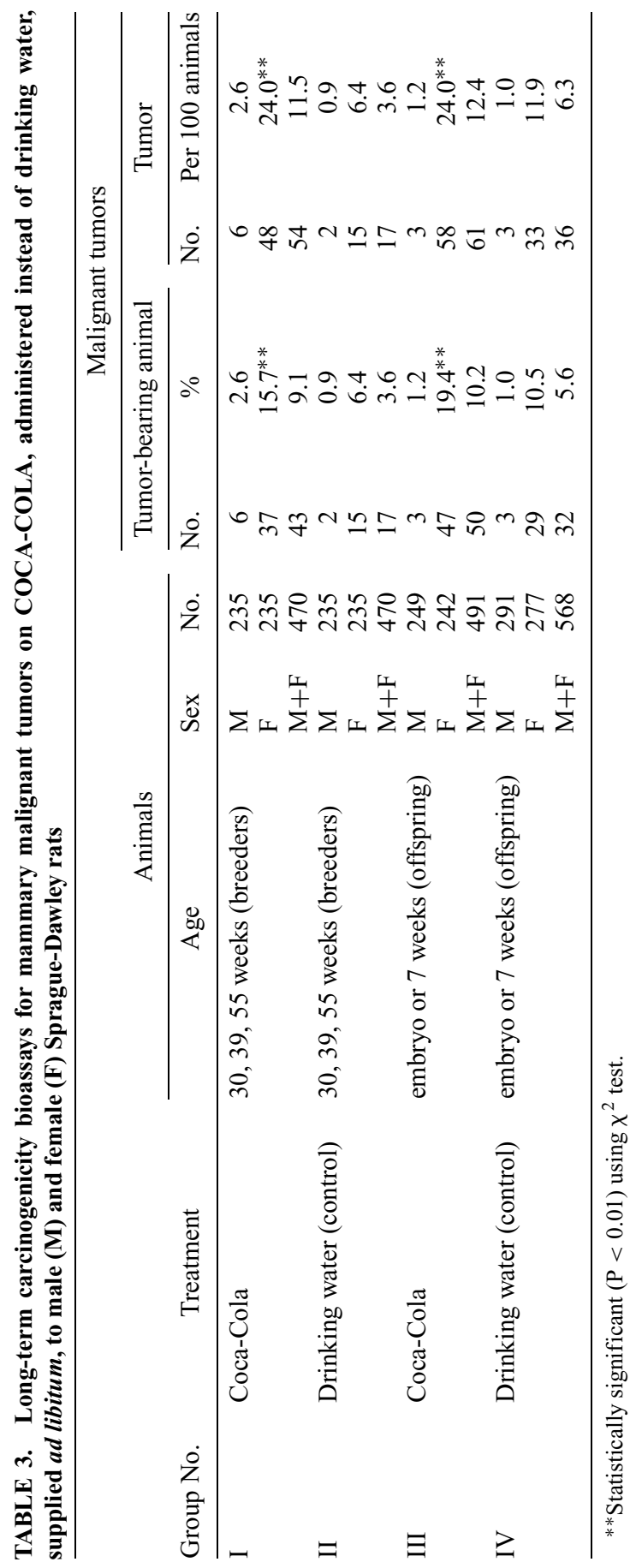




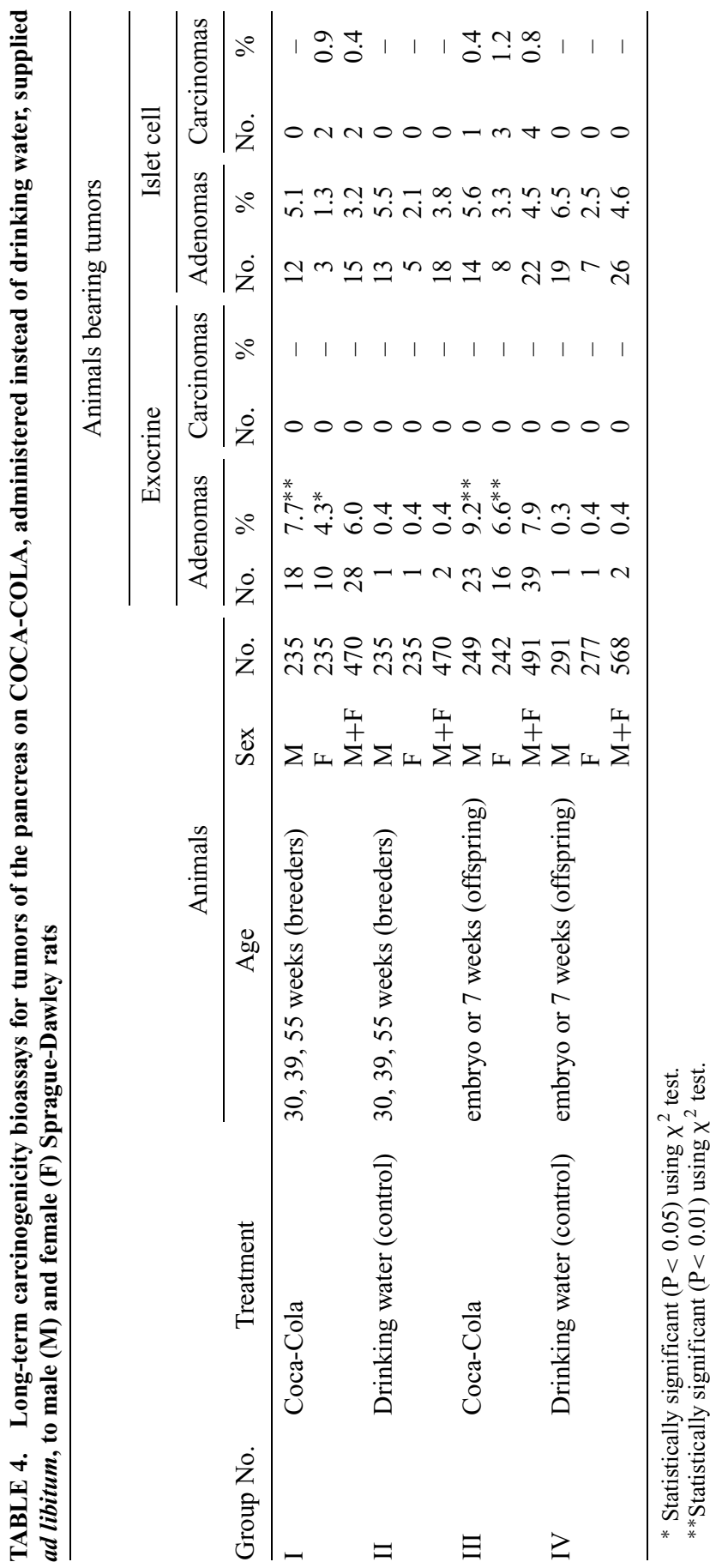


carcinogenesis bioassay has been conducted to evaluate the beverage mixture for its potential carcinogenic effects.

The results of the study conducted at the CMCRC/ERF have shown the following:

1. an increase of fluid consumption in all animals, both breeders and offspring, administered Coca-Cola as a substitute for drinking water and a general decrease in food consumption;

2. an increase in body weight in all treated animals.

When compared to controls, animals treated with Coca-Cola also demonstrated the following oncological effects:

1. a statistically significant increase of the incidence in females, both breeders and offspring, bearing malignant mammary tumors. In our opinion, this observation confirms the correlation between the increase in body weight and an increased risk of mammary cancer;

2. a statistically significant increase in the incidence of exocrine adenomas of the pancreas in both male and female breeders and offspring;

3. an increased incidence, albeit not statistically significant, of pancreatic islet cell carcinomas in females. Because of the rarity of pancreatic iselt cell carcinomas in our historical controls, the biological significance of the increased incidence of this malignant tumor cannot be underestimated.

Although humans do not consume this beverage under the same conditions designed in our experiment, the results nevertheless confirm that an exaggerated ingestion of high caloric beverages, such as regular soft-drinks, can lead to a marked increase in body weight which in turn presents an increased risk for developing cancer.

\section{ACKNOWLEDGMENTS}

We thank the research staff of the CMCRC/ERF and Kathryn Knowles for her support in the preparation of the manuscripts.

\section{REFERENCES}

1. Tannenbaum, A. 1940. The initiation and growth of tumors. I: effects of underfeeding. Am. J. Cancer 38: 335-350.

2. Tannenbaum, A. \& H. Silverstone. 1953. Nutrition in relation to cancer. Adv. Cancer Res. 1: 451-501.

3. CALLE, E. et al. 2003. Overweight, obesity, and mortality from cancer in a prospectively studied cohort of U.S. Adults. N. Engl. J. Med 348: 1625-1638. 
4. RAPP, K. et al. 2005. Obesity and incidence of cancer: a large cohort study of over 145000 adults in Austria. Br. J. Cancer 93: 1062-1106.

5. SoffritTi, M. et al. 1999. Mega-experiments to identify and assess diffuse carcinogenic risks. Ann. N. Y. Acad. Sci. 895: 43-55.

6. SOFFrITTI, M. et al. 1996. Results of experimental bioassays on the chemopreventive effects of vitamin A and N-(4-hydroxyphenyl)-retinamide (HPR) on mammary cancer. In The Scientific Bases of Cancer Chemoprevention. C. Maltoni, M. Soffritti \& W. Davis, Eds.: International Congress Series 1120 : 241-248. Elsevier. Amsterdam, The Netherlands.

7. The Coca-Cola Company Annual Report. 2005. http://www.cocacola.com [accessed 16 May 2006] 